\title{
The Speed of Light and Uncertainty Principle of the Macro-world
}

\author{
Gocho V. Sharlanov ${ }^{1}$ \\ ${ }^{1}$ Stara Zagora, Bulgaria 6014, EU \\ Correspondance: Gocho V. Sharlanov, str. 22 Gen. Ivan Pashinov, entr. V, apt. 63, Stara Zagora, Bulgaria 6014, EU. Tel: \\ 359-894-853-960. E-mail: gsharlanov@yahoo.com, gsharlanov@engineer.bg
}

Received: June 19, 2012 Accepted: July 6, 2012 Online Published: October 26, 2012

doi:10.5539/apr.v4n4p118 URL: http://dx.doi.org/10.5539/apr.v4n4p118

\begin{abstract}
The facts are:

- The speed of light is different in different directions in the reference system connected with the Earth's surface, moving with $0.46 \mathrm{~km} / \mathrm{s}$ at the equator. It has been demonstrated in various experiments for almost a hundred years.

- There is no experiment carried out in the area near the Earth's surface (our local reality), that proves ether-drift result on the speed of light, due to the Earth's motion on its trajectory around the Sun, which is approximately $30 \mathrm{~km} / \mathrm{s}$.

- Undeniable fact is that the speed of light in a reference system, connected with the space itself, is constant in a homogeneous gravitational field, or in local time-spatial domain with the same gravitational potential.

- The speed of the light in a reference system, connected with the space itself, changes with the change of the intensity of the gravitational field. The Shapiro-time-delay effect and the anomaly in acceleration of the spaceships "Pioneer-10/11", "Galileo", and "Ulysses" at the border of the Solar system - are actually proving experiments of this reality.

In this paper, it is shown why in a local time-spatial domain, where the units of the time and space are defined, it is impossible to register the change of the speed of light in a reference system connected with the space itself. It is because of the way of definition of the units of time and length and because of the synchronous change of all local units, all local physical constants-of the entire physical reality. As a conclusion - "uncertainty principle of the macro-world" is proposed.
\end{abstract}

Keywords: speed of light, uncertainty principle, Special Relativity, General Relativity, SI units, postulate, light propagation, electromagnetic radiation

\section{Introduction}

"The formulation of a given problem is far more essential, than its solution that could be simply a question of mathematical or experimental skills". (Albert Einstein, Leopold Infeld, "Evolution of Physics", 1938)

Let's see the definitions of the Base Units "metre" and "second" in International System of Units "SI".

\subsection{About the Definitions-"SI Base Units in the Framework of General Relativity"}

Overall, the definitions of the units in "SI" in the framework of general relativity are written in (BIPM SI brochure $8^{\text {th }}$ ed., 1.5 SI units in the framework of general relativity, 2006):

"The definitions of the base units of the SI were adopted in a context that takes no account of relativistic effects. When such account is taken, it is clear that the definitions apply only in a small spatial domain sharing the motion of the standards that realize them. These units are known as proper units; they are realized from local experiments in which the relativistic effects that need to be taken into account are those of special relativity."

1.2 Definitions of the Base Units "Second" and "Metre" (of Time and Length) in the International System of Units "SI"

- "Second", the SI base unit of time.

The (13 ${ }^{\text {th }}$ meeting of the CGPM Resolution $\left.1,1967 / 68\right)$ replaced the definition of "the second" by the following: 
"The second is the duration of 9192631770 periods of the radiation corresponding to the transition between the two hyperfine levels of the ground state of the caesium 133 atom."

At its 1997 meeting, the BIPM affirmed that: "This definition refers to a caesium atom at rest at a temperature of $0^{0} \mathrm{~K} . "$

- "Metre", the SI base unit of length.

The definition of "metre" since 1889, based on the international Prototype of platinum-iridium, is abrogated ( $11^{\text {th }}$ meeting of the CGPM, Resolution 6, 1960). The definition of "the metre" was replaced by the following:

"The metre is the length equal to 1650763.73 wavelengths in vacuum of the radiation corresponding to the transition between the levels $2 p_{10}$ and $5 d_{5}$ of the krypton 86 atom."

With Resolution 1 (17th meeting of the CGPM, 1983) "the metre" is replaced as:

"The metre is the length of the path traveled by light in vacuum during a time interval of 1/299 792458 of a second."

This definition is based on the postulate "Invariance of $\mathrm{c}$ (speed of light)". The symbol $\mathbf{c}_{\mathbf{0}}$ (or sometimes simply c), is the conventional symbol for the speed of light in vacuum.

The value of the speed of light is recommended with Resolution 2 (15th meeting of the CGPM, 1975): "[CGPM] recommends the use of the resulting value for the speed of propagation of electromagnetic waves in vacuum $c=$ 299792458 metres per second."

\subsection{The Facts about the Speed of Light}

The Special Theory of Relativity applies to homogeneous field without gravity and is based on the principle of relativity and the postulate of the constancy of the speed of light.

"We will raise this conjecture (the purport of which will hereafter be called the "Principle of Relativity") to the status of a postulate, and also introduce another postulate, which is only apparently irreconcilable with the former, namely, that light is always propagated in empty space with a definite velocity $c$ which is independent of the state of motion of the emitting body" (Einstein, 1905).

- In this definition Einstein says nothing about the influence of the motion of the Observer's reference system on the measured speed of light. It is refuted a long ago that the speed of light is independent from the movement of the Observer's reference system. The dependence of the measured speed of light is demonstrated in various experiments by (Sagnac, 1914; Michelson \& Gale, 1925; Miller, 1933; Marmet, 2000; Ashby, 2003; Kelly, 2005; Gift, 2010). They prove that the measured speed of light is different in different directions in the reference system associated with the moving Earth's surface with the linear velocity of $1,669.72 \mathrm{~km} / \mathrm{hr}$ at the equator. But the speed of light is constant in the reference system associated with the space itself, where the light propagates (Sharlanov, 2011). All of these experiments are carried out in the time-spatial domain with homogenous gravitational field - in the area near the Earth's surface. Today the GPS utilizes a clock-synchronization procedure based on invariance of $\boldsymbol{c}$ in vacuum and "Sagnac correction". This procedure is developed on the base of published standards and synchronization rules.

Note: Therefore the speed of light should be defined in the coordinate system associated with the space, not in the coordinate system associated with the Earth's surface. One of the methods, by which the speed of light can be defined in the coordinate system associated with the space, is by taking the arithmetical average of the measured velocities in two opposite directions (East-West) in the coordinate system associated with the Earth's surface. This note is directed to the current definition of unit "meter" by means of the speed of light and gives a proposal for a more accurate measurement of "the speed of light in vacuum" - $\left(15^{\text {th }}\right.$ meeting of the CGPM Resolution 2, 1975).

So the reader should be aware that in this paper the behavior of light is concerned only in the frame of reference connected to the space itself, where the light emits, absorbs and spreads. In this reference system the speed of light is constant and it is a fact only if the spatial area is in homogeneous gravitational field, in other words with equal gravitational potential, or with equal level of contraction/expansion of the space-time.

- Another fact we should be aware of:

"For experiments upon the Earth tell us nothing of the fact that we are moving about the Sun with a velocity of approximately 30 kilometres per second ... But all experiments have shown that electromagnetic and optical phenomena, relatively to the Earth as the body of reference, are not influenced by the translational velocity of the Earth." (Einstein, 1922). 
Or, Einstein asserted and it is true, that being on the Earth's trajectory around the Sun, we cannot find reference system in our local time-spatial domain (near the Earth's surface), where we can register any change of the speed of light because of the moving of the Earth around the Sun. Or, we cannot register any change neither because of the different directions of the Earth's motion around the Sun, nor because of the different "translational velocity of the Earth" at its trajectory (min. orbital velocity is $29.3 \mathrm{~km} / \mathrm{s}$; max. orbital velocity is $30.3 \mathrm{~km} / \mathrm{s}$.)

\subsection{The Changing Local Units}

Our units are only for our local "small spatial domain" (BIPM SI brochure $8^{\text {th }}$ ed., 2006), or our units are local units. Furthermore, the level of contraction/expansion of the space-time continuously changes in each local time-spatial domain (the Universe is not static), but it cannot be measured and understood.

Note: Often, the term "gravitational potential" is used instead of the term "intensity of gravitational field". In the present paper it is accepted that a higher "gravitational potential" corresponds to lower "intensity of gravitational field" or to weaker gravitational field.

This article discusses:

- How the speed of light in vacuum (in the reference system associated with the space itself) changes with the change of the gravitational potential (or with the change of the level of expansion/contraction of the space-time).

- Why the change of the speed of light in the reference system associated with the space itself cannot be determined (or true values of that change cannot be obtained, as a result of measurement carried out at a certain time). As a whole, in this article it is presented that the change of the speed of light cannot be determined in the local spatial domain, where the SI based units of time and length have been defined and where the experiment is carried out.

- As a logical consequence of this discussion: "Uncertainty principle of the macro-world" is a proposed.

2. The Postulate "Invariance of the Speed of Sight" - the Both Two Reasons, That Make Impossible to Be Determined the Change of the Speed of Light

Coexistence of two reasons makes impossible to determine the change in the speed of light (see below).

\subsection{Definitions of the SI Base Units "Metre" and "Second" by Means of an Identical Experiment - by Means of} the Same "Standard Radiation"

Actually, the definition of a unit means that the exact definite experiment is carried out at exact definite initial conditions. As a result of this experiment, a certain "number" is fixed which is defined as "unit" and is given a name.

No discrepancy and/or contradictions will be encountered, if we accept, that the SI base units of the time and length are defined as a result of an experiment on the base of one definite electromagnetic radiation:

Convention: Let's name this radiation in the article as "standard radiation", and $v_{\mathrm{s}}$ and $\lambda_{\mathrm{s}}$ are the frequency and wavelength of this "standard radiation".

This way of defining the SI base units "second" and "metre" is convenient for easier understanding of the main idea by readers, when the units defined in time-spatial areas (domains) with different gravitational potential are comparing. Let's accept, the "standard radiation" is: "the radiation corresponding to the transition between the two hyperfine levels of the ground state of the caesium 133 atom" - and $\nu_{\mathrm{s}}$ and $\lambda_{\mathrm{s}}$ are the frequency and wavelength of this "standard radiation". In this way the definition of the unit of time "second" is the same as adopted by BIPM and we can define the unit of length "the metre" as: "the length, equal to 30.66331899 wavelengths of the radiation corresponding to the transition between the two hyperfine levels of the ground state of the caesium 133 atom in a vacuum, at rest, at a temperature of $0^{\circ} \mathrm{K}$."

In this way, if for our "standard radiation" the number of periods per one "second" is exactly $\mathrm{N}_{\mathrm{s}}=9,192,631,770$; the number of wavelengths per one "metre" is exactly $\mathrm{N}_{\mathrm{m}}=30.66331899$ - and the local constant "speed of light" will have the exact value (number):

$$
c=N_{s} / N_{m}=9,192,631,770 / 30.66331899=299,792,458[\mathrm{~m} / \mathrm{s}]
$$

\section{2 (Well-known) Consequences}

In his work "On the Influence of Gravitation on the Propagation of Light" (Einstein, 1911), Albert Einstein considers the change of the frequency of light in places with different gravitational potential: 


$$
v=v_{0}\left(1+\frac{\Phi}{c^{2}}\right)
$$

Here $v$ is the frequency in a location with a gravitational potential $\Phi$, relative to the origin of the co-ordinates; $v_{0}$ is the frequency in the origin of the co-ordinates; and the speed of light $c$ is a constant. However, the change of the wavelength (and therefore a meter) is not taken into account when calculating $\Phi$. Further in this article, Einstein states:

"If we call the speed of light at the origin of co-ordinates $c_{0}$, then the speed of light c at a place with the gravitation potential $\Phi$ will be given by the relation:

$$
c=c_{0}\left(1+\frac{\Phi}{c^{2}}\right)
$$

The principle of the constancy of the speed of light holds good according to this theory in a different form from the one that usually underlies the ordinary theory of relativity."

The change of the unit "meter", as was mentioned above, is not taken into consideration by Einstein in the equations (2) and (3). If this change was set into the expressions of the " $\Phi$ " and "c" - the result would have been uncertainty (see below "6. Uncertainty principle of the macro-world").

We should be aware that not only the frequency and the speed of light - the entire electromagnetic spectrum changes all its parameters and characteristics in full synchrony with the contraction/expansion of the space-time. It means that any atom emits photons (radiation with frequency, wavelength and speed) in synchrony with the space-time distortion. This explains the registered anomaly in acceleration of the spaceships "Pioneer-10/11", "Galileo", and "Ulysses" at the border of the Solar system, which is actually proving experiment of this hypothesis: "At the emission, spreading and absorption of any electromagnetic radiation, its frequency, wavelength and speed are in synchrony with the intensity of the gravitational field, with the space-time distortion (curvature) of the "space-time domains" where the light emits, passes or absorbs."

Let's examine the following two scenarios (A and B):

"A"-scenario) At taking off of the electromagnetic radiation from the surface of a star and entering interstellar space (weaker gravitational field):

- the time goes faster, which is consequence of increasing the frequency $v$ of the electromagnetic radiation - the "second" becomes shorter, because "the duration of 9192631770 periods of the radiation corresponding to the transition between the two hyperfine levels of the ground state of the caesium 133 atom" is shorter."

- the "meter" becomes longer, because "the length, equal to 30.66331899 wavelengths of the radiation corresponding to the transition between the two hyperfine levels of the ground state of the caesium 133 atom" is longer (the wavelength $\lambda$ becomes longer because of the spatial expansion).

- $\quad$ Therefore, the speed of light increases $(c=\lambda v)$.

"B"-scenario) Vice-versa - when approaching the Earth's surface (towards stronger gravitational field):

- the time goes slower, which is a consequence of the frequency decreasing of the electromagnetic radiation. In other words, the defined by means of the same experiment unit of time "second" becomes longer;

- the "meter" becomes shorter (the wavelength of the electromagnetic radiation decreases).

- $\quad$ Therefore, the speed of light decreases $(c=\lambda v)$. 


\subsection{The First Reason Why the Change of the Speed of Light Cannot Be Determined: "The Way of Definition" of the SI Base Units of Length and Time - the "Metre" and the "Second")}

The case under consideration:

\subsubsection{Initial Conditions}

(i) Let's look at two local small time-spatial areas (domains) - one is $\mathrm{D}_{1}$, and the other is $\mathrm{D}_{2}$, in the real warped space-time of our Universe. Let them generally have different gravitational potential (different level of contraction/expansion of the space-time), and the gravitational potential of $\mathrm{D}_{2}$ is higher than gravitational potential of $\mathrm{D}_{1}$, or $\left(\Phi_{2}>\Phi_{1}\right)$. It means, to higher "gravitational potential" corresponds lower "intensity of gravitational field".

(ii) The SI base units - "second" and "meter" are defined in $\mathrm{D}_{1}$ and $\mathrm{D}_{2}$ by means of the same experiment (under 2.1.) - using ,the standard radiation", corresponding to the transition between the same two hyperfine levels of the Cesium-133 atom.

In this way by fixing the same numbers $\mathrm{N}_{\mathrm{s}}$ and $\mathrm{N}_{\mathrm{m}}$, the units of the length and time in the two domains with $\left(\Phi_{2}>\Phi_{1}\right)$ will be different, because the frequency and the wavelength are different.

\subsubsection{Analysis}

Convention: The subscript indicates in what domain the measurement is carried out and the superscript indicates the domain where the unit is defined.

Let us name the unit of time, obtained by measuring the duration of $\mathrm{N}_{\mathrm{s}}=9192631770$ periods of "the standard radiation" in $\mathrm{D}_{1}$ "second-1", and in $\mathrm{D}_{2}$ - respectively "second-2". Obviously "second-1" will be with longer duration than "second-2", because the frequency of "the standard radiation" in $\mathrm{D}_{2}$ is higher than in $\mathrm{D}_{1}$. It is because the gravitational field in $D_{1}$ is stronger, or the gravitational potential of $D_{2}$ in relation to $D_{1}$ is $\left(\Phi_{2}>\Phi_{1}\right)$. Or the time in $\mathrm{D}_{1}$ passes more slowly.

Let us name the unit of length, obtained by measuring the length of $\mathrm{N}_{\mathrm{m}}=30.66331899$ wavelengths of "the standard radiation" in $\mathrm{D}_{1}$ "metre-1", and in $\mathrm{D}_{2}$ - respectively "metre-2". Obviously "metre-2" will be longer than "metre-1", because the wavelength of "the standard radiation" in $\mathrm{D}_{2}$ is longer than in $\mathrm{D}_{1}$. It is because the gravitational potential of $\mathrm{D}_{2}$ in relation to $\mathrm{D}_{1}$ is $\Phi_{2}>\Phi_{1}$ - or the space in $\mathrm{D}_{2}$ is more expanded than the space in $\mathrm{D}_{1}$, or the gravitational field in $\mathrm{D}_{2}$ is weaker.

The results are:

- Measuring the frequency and the wavelength of "the standard radiation" in domain $\mathrm{D}_{1}$, but with the units "second-2" and "metre-2", defined in domain $\mathrm{D}_{2}$, where $\Phi_{2}>\Phi_{1}$ " - let us mark them as ${v_{\mathrm{s} 1}}^{2}$ and $\lambda_{\mathrm{s} 1}{ }^{2}$ :

If we can measure the frequency and the wavelength of ,the standard radiation" in $\mathrm{D}_{1}$ with the units defined in $\mathrm{D}_{2}$ (where "the metre-2" is longer and "the second-2" is shorter than those in $\mathrm{D}_{1}$ ), the values (results) will turn out to be smaller than those measured with their own units defined in time-spatial domain $\mathrm{D}_{1}$ :

$$
v_{s I}{ }^{2}<v_{s I}{ }^{l} \text { and } \lambda_{s 1}{ }^{2}<\lambda_{s l}{ }^{l}
$$

In other words, within the shorter "second-2" we will count less number of periods $\mathrm{N}_{\mathrm{s}}$, than if we count within the longer "second-1" $\left(v_{\mathrm{s} 1}^{2}<v_{\mathrm{s} 1}{ }^{1}\right)$ and the value of the wavelength of "the standard radiation" in $\mathrm{D}_{1}$, measured with longer "meter-2" of $\mathrm{D}_{2}$ will turns out to be smaller than if we measured it with the own unit in $\mathrm{D}_{1}$ "meter-1" $-\left(\lambda_{\mathrm{s} 1}{ }^{2}<\lambda_{\mathrm{s} 1}{ }^{1}\right)$.

The resulting value (number) of the mental experiment "measurement of the speed of light in domain $\mathrm{D}_{1}$ with the units defined in $\mathrm{D}_{2}$ " (the real experiment is not possible to be carried out), will be much less if we were using the units defined within the domain $\mathrm{D}_{1}$ :

$$
c_{1}{ }^{2}=v_{s l}{ }^{2} \cdot \lambda_{s l}{ }^{2}<<v_{s l}{ }^{l} \cdot \lambda_{s l}{ }^{l}=c_{l}{ }^{l}=299,792,458[\mathrm{~m} / \mathrm{s}]
$$

- Measuring the frequency and the wavelength of the „standard radiation” in domain $\mathrm{D}_{2}$, but with the units "second-1" and "metre-1" defined in domain $\mathrm{D}_{1}$, where $\Phi_{1}<\Phi_{2}-$ let us mark them as $v_{\mathrm{s} 2}{ }^{1}$ and $\lambda_{\mathrm{s} 2}{ }^{1}$ :

Respectively, if we can measure the frequency and the wavelength of "the standard radiation" in $\mathrm{D}_{2}$ with the units defined in $\mathrm{D}_{1}$ (where "the metre-1" is shorter and the unit "second-1" is longer than those in $\mathrm{D}_{2}$ ), the values (results) will turn out to be higher than those measured with their own units defined in time-spatial domain $\mathrm{D}_{2}$ :

$$
v_{\mathrm{s} 2}{ }^{1}>v_{\mathrm{s} 2}{ }^{2} \text { and } \lambda_{\mathrm{s} 2}{ }^{1}>\lambda_{\mathrm{s} 2}{ }^{2}
$$

And respectively, when the measurement of the speed of light in domain $\mathrm{D}_{2}$ is made with the units defined in $\mathrm{D}_{1}$, 
the resulting value (number) will be much bigger if we were using the units defined within the domain $\mathrm{D}_{2}$ :

$$
c_{2}{ }^{1}=\lambda_{s 2}{ }^{l} \cdot v_{s 2}{ }^{l}>>\lambda_{s 2}{ }^{2} \cdot v_{s 2}{ }^{2}=c_{2}{ }^{2}=299,792,458[\mathrm{~m} / \mathrm{s}]
$$

Summary: Therefore, measuring the speed of light in two domains with different gravitational potentials, respectively $\Phi_{1}$ of $D_{1}$ and $\Phi_{2}$ of $D_{2},\left(\Phi_{2}>\Phi_{1}\right)$, but with the units defined in only one of domains - the much greater speed of light in $\mathrm{D}_{2}$ will be confirmed in the two above mentioned points of view (the two cases of measuring - (5) and (7)).

But if someone measures the frequency $v_{\mathrm{s}}$ and the wavelength $\lambda_{\mathrm{s}}$ of "the standard radiation" in $\mathrm{D}_{1}$ and in $\mathrm{D}_{2}$ with their own units (defined in their time-spatial domains), he will obtain the same value.

$$
v_{s 1}{ }^{1}=v_{s 2}{ }^{2} \text { and } \lambda_{s 1}{ }^{1}=\lambda_{s 2}{ }^{2}
$$

although the frequency and the wavelength of "the standard radiation" in $\mathrm{D}_{1}$ are less than in $\mathrm{D}_{2}$.

Respectively, if someone measures speed of light $\mathrm{c}_{1}$ in domain $\mathrm{D}_{1}$, and $\mathrm{c}_{2}$ in domain $\mathrm{D}_{2}$ with their own units defined in their time-spatial domains, he will obtain the same value (will have the same number), or:

$$
\mathbf{c}_{1}{ }^{1}=\mathbf{c}_{2}{ }^{2}=\mathrm{N}_{\mathrm{s}} / \mathrm{N}_{\mathrm{m}}=9,192,631,770 / 30.66331899=299,792,458[\mathrm{~m} / \mathrm{s}]
$$

And all of this is in spite of the fact, that the units "metre" and "second" in the two domains are different and the speed of light is different too.

\subsubsection{Summary of the Analysis}

- In spite of the fact that the SI base units ("metre" and "second") are different in two domains with different gravitational potential (or with different level of contraction/expansion), the results of the measurement of the frequency and wavelength of "the standard radiation" into the two domains will be the same. This is because the SI base units ("metre" and "second") are defined in these two domains by means of the electromagnetic radiation, on the base of identical experiments.

- The difference of the speed of light cannot be registered into the domains with different gravitational potential (or with different level of contraction/expansion), as well as the change of the speed of light in the same domain, when the level of contraction/expansion of the domain is changing. It is because the change of the SI base units ("second" and "metre") cannot be determined.

- Further more, if we choose another "standard radiation"- different from the previous electromagnetic radiation, "the radiation corresponding to the transition between the two hyperfine levels of the ground state of the caesium 133 atom", and if we define the SI base units of time and length ("the second" and "metre") through the same experiment, (fixing the same numbers $\mathrm{N}_{\mathrm{s}}$ and $\mathrm{N}_{\mathrm{m}}$ ) - we will measure the same number/result for the speed of light (1) in spite of the fact, that the units "metre" and "second" are different.

Summary: The change of speed of light cannot be registered in case of changing the gravitational potential (or in case of changing the level of contraction/ expansion) of the time-spatial domain where the Observer is located and where the units are defined.

2.4 Second Reason Why the Change of the Speed of Light Cannot Be Determined: The Synchronous Change of All Local Constants and All SI Base and Derived Units (of the Entire Physical Reality) at Changing the Level of Contraction/Expansion of the Space-time

\subsubsection{Awareness of the Electromagnetic Radiation as a Vibration of the Space-time Itself}

The space-time itself is often called a vacuum. The space-time exists on many levels - it is among the elementary particles of the matter, among all the planets, stars and galaxies. All these levels are mutually interconnected, depending on each other, and changing in full and perfect synchrony and harmony. The space-time is curved, bended and warped at a micro and at a macro-level - curved, bended and warped by the smallest particles of matter (like nucleus of the atom), by the planets, stars, galaxies... in all areas of the Universe.

The electromagnetic radiation originates in the space itself as a vibration generated at the transition of the electrons from one level of space and energy to another, in conformity with the local level of the contraction/expansion of the space-time. The electromagnetic radiation spreads in the space-time, vibrating again in synchrony with the local level of the contraction/expansion of the space-time ... and is absorbed (at the transition of electrons from one level of space and energy to another, giving energy to electrons again in full conformity with the local level of the contraction/expansion of the space-time). Therefore, we should realize that all spectrum of electromagnetic radiation is a type of vibration of the space-time itself, and all the electromagnetic spectrum changes its parameters and characteristics in full synchronization with the contraction/expansion of the 
curved, bended and warped space-time. That's why, there is no experiment carried out in the area near the Earth's surface that proves ether-drift result on the speed of light, due to the Earth's motion on its trajectory around the Sun. On one hand, this synchrony means that the electromagnetic radiations are oppressed (overwhelmed) in a strong gravitational field (at a high level of contraction of the space-time). They transform themselves into vibrations with shorter wavelengths and with lower frequencies, which means lower speed of spreading $(\mathrm{c}=\lambda v)$. On the other hand, when the electromagnetic radiation enters into weak gravitational field (higher level of expansion of space-time), the wavelengths of electromagnetic vibrations become longer and frequencies become higher, which means a higher speed of spreading $(c=\lambda v)$. The Shapiro-time-delay effect (Shapiro, Irwin I., 1964) and the anomaly in acceleration of spaceships "Pioneer-10/11", "Galileo", and "Ulysses" at the border of the Solar system - are actually a proving experiments of this hypothesis (Sharlanov, 2011).

2.4.2 Awareness of the Speed of Light as a Coefficient of Correlation between the Wavelength and Frequency of Any Electromagnetic Radiation in a Time-Spatial Domain with a Certain Level of Contraction/Expansion

In other words, the wavelength and the frequency of any electromagnetic radiation are mutually connected with a coefficient of correlation which we call "the speed of light". This coefficient is a local constant for any time-spatial domain with certain level of contraction/expansion of the space-time. This local constant (the speed of light) is different for the time-spatial domains with different level of contraction/expansion, but it is not possible to determine this difference or change. The measured value of this coefficient is always the same, because the units of time and length are defined through the changing wavelength and frequency of the electromagnetic radiation.

Not only the speed of light, but all the local constants and all the based and derived units (all the local units) synchronously change with the changing of the level of contraction/expansion of space-time. It means a synchronous change of the entire Physical Reality.

\section{Conclusions}

Conclusion 1: In the frame of reference associated with the space itself, where the light is actually spreading - it is not possible to prove by measurement, carried out at a certain time, (getting true and valid values as a result of this measurement) - that there is a change of the value of the speed of light in the time-spatial domain where the based units of time and length are defined.

This is the result of the above mentioned two reasons:

- The way of definition of the SI base units ("metre" and "second"), based on identical experiments, through interrelated characteristics of the electromagnetic radiation;

- The synchronous change of the entire physical Reality at changing the level of contraction/expansion of the space-time.

Furthermore, indeterminacy is not only refer to the change of the constant "speed of light":

Conclusion 2: In the local "time-spatial domain", where physical units are defined, it is not possible to prove by measurement the change of the value of any physical constant (the speed of light, Planck's constant, etc.).

Conclusion 3: Another conclusion is that 'the 'ether' is considered to be the 'warped space-time of the Universe' itself" (Sharlanov, 2011). The electromagnetic field is upon, over the based gravitational space-time field. That's why, in the frame of reference connected with the space itself, there is no experiment carried out in our local reality that proves ether-drift result on the speed of light, due to the Earth's motion on its trajectory around the Sun, which is approximately $30 \mathrm{~km} / \mathrm{s}$.

\section{Uncertainty Principle of the Macro-world}

Instead of the postulate Invariance of $c$ - the Uncertainty principle for the macro-world should be approved:

The uncertainty of the macro-world consists in the fact, that we cannot measure or calculate in our local time-spatial domain (where the units are defined), neither the change of the defined by us units, nor the change of all our local constants, because they all change in perfect synchrony with the change of the entire physical reality. Also, we cannot measure or calculate any change in the entire physical reality in another remote time-spatial domain with different level of contraction/expansion of the space-time, because the units in the remote domain are uncertainly different.

Some consequences are:

The accuracy of the measurement or calculation (even in theory) of the DISTANCE to a remote "time-spatial domain" (or to an object in the Universe), as well the accuracy of the measurement or calculation (even in theory) of the TRAVEL TIME OF THE LIGHT from a "time-spatial domain" (or an object in the Universe), is in inverse 
proportion:

- to the remoteness

- to the relative level of contraction/expansion of the other "time-spatial domain" in comparison with that of the Observer.

The remoteness introduces inaccuracy of the information at its transmission (a shift due to the change of the unit of the time while the information is transmitting).

The relative level of contraction/expansion between "time-spatial domains" of the Object and Experimenter introduces inaccuracy of the information due to the uncertain difference of the base units of time and length between the "time-spatial domains".

As epilogue:

The global uncertainty is due to the nature of space and time, which are intimately and mutually connected with each other in all the micro- and macro- levels of the curved, bended and warped space-time of the Universe.

This is also the "Border of the Experimental Provability"...

\section{References}

$11^{\text {th }}$ meeting of the CGPM (1960) Resolution 6. Retrieved from http://www.bipm.org/en/CGPM/db/11/6/

$13^{\text {th }}$ meeting of the CGPM (1967/68) Resolution 1. Retrieved from http://www.bipm.org/en/CGPM/db/13/1/

$15^{\text {th }}$ meeting of the CGPM (1975) Resolution 2. Retrieved from http://www.bipm.org/en/CGPM/db/15/2/

$17^{\text {th }}$ meeting of the CGPM (1983) Resolution 1. Retrieved from http://www.bipm.org/en/CGPM/db/17/1/\#notes

Ashby, N. (2003). Relativity in the Global Positioning System. Living Reviews in Relativity, 6, 1. Retrieved from http://www.ipgp.fr/ tarantola/Files/Professional/GPS/Ashby_2003.pdf

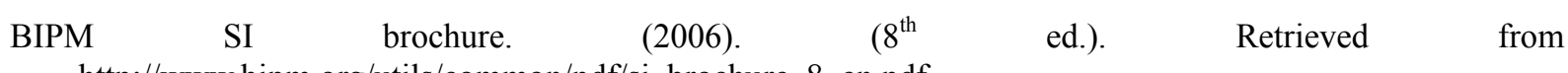
http://www.bipm.org/utils/common/pdf/si_brochure_8_en.pdf

Einstein, A. (1905). On The Electrodynamics of Moving Bodies. Annalen der Physik scientific journal, p.1. Retrieved from http://www.fourmilab.ch/etexts/einstein/specrel/specrel.pdf

Einstein, A. (1911). On the Influence of Gravitation on the Propagation of Light. Annalen der Physik [35]. Retrieved from http://qss.stanford.edu/ godfrey/physics/Einstein_On_the_Influ_of_Grav_on_Light.pdf

Einstein, A. (1922). The meaning of of Relativity (5th ed., 1974). Princeton, NJ: Princeton University Press.

Gift, S. J. G. (2010). One-Way Light Speed Determination Using the Range Measurement Equation of the GPS. Applied Physics Research, 3(1), 110. http://dx.doi.org/10.5539/apr.v3n1p110

Kelly, A. (2005). Challenging Modern Physic. Florida: BrownWalker Press.

Marmet, P. (2000). The GPS and the Constant Velocity of Light, Acta Scientiarum, 22, 1269.

Michelson, A. A., \& Gale, H. E. (1925). Effect of the Earth's Rotation on the Velocity of Light, Astrophysical J., 61, 140. http://dx.doi.org/10.1086/142879

Miller, D. C. (1933). The Ether-Drift Experiment and the Determination of the Absolute Motion the Earth, Reviews of Modern Physics, 5. http://dx.doi.org/10.1103/RevModPhys.5.203

Sagnac, Georges. (1913). On the proof of the reality of the luminiferous aether by the experiment with a rotating interferometer. Comptes Rendus, 157, 1410-1413. http://dx.doi.org/10.1103/PhysRevLett.13.789

Shapiro, Irwin I. (1964). Fourth Test of General Relativity. Physical Review Letters, 13(26), 789-791. Retrieved from http://en.wikisource.org/wiki/On_the_Proof_of_the_Reality_of_the_Luminiferous_Aether

Sharlanov, G. V. (2011). The Influence of Gravitation on the Speed of Light and an Explanation of the Pioneer 10\&11 Acceleration Anomaly. Applied Physics Research, 3(2), 241. http://dx.doi.org/10.5539/apr.v3n2p241 